\section{Spiral Computed Tomography with Phytocomposition as a Diagnostic Tool for Adhesive Intestinal Obstruction}

\author{
Mufazalov F. ${ }^{1 \oplus}$, Sufiyarov I. ${ }^{2}$, Hasanov A. ${ }^{2}$, Yamalova G. ${ }^{3}$, \\ Bakirov E. ${ }^{3}$, Samorodov A. ${ }^{4 * \odot}$
}

\begin{abstract}
Background: Adhesive intestinal obstruction is a common and potentially lethal complication after surgical interventions in the abdomen. Radiologic imaging is the main diagnostic method.
\end{abstract}

Objective: This study aims to analyse the diagnostic value of spiral computed tomography with a novel method $(\mathrm{n}=54)$.

Material and Methods: In this multidirectional cohort study, we present the data with non-parallel (historical) control. This study included the analysis of results of patients with a diagnosis of intestinal obstruction $(n=54)$ who were admitted to the surgical departments of the City Clinical Hospitals (Ufa city) from 2013 to 2019; the patients' examination methods included computed tomography with conventional enhancement. The proposed novel enhancement method was implemented by ingesting a mixture containing $50 \mathrm{ml}$ of the contrast Unigexol $(300 \mathrm{mg})$ in $1.0 \mathrm{~L}$ cold mineral carbonated water, and Computed tomography (CT) was performed during 40 min after ingesting the contrast meal. Further, the patients with suspected obstruction in the colon were administered a pre-prepared contrast enema with a decoction of leaves of smoke-tree (100 g), chamomile flowers (100 g) and calendula flowers (100 g). Additionally, CT was performed.

Results: Obstruction was conservatively stopped in 24 (44.4\%) patients of the main group. Remaining 30 (55.6\%) patients from the main group were operated with minimal surgical access in the early stages.

Conclusion: Owing to early diagnosis of intestinal obstruction and application of the phytocomposition during the examination, exerting various effects such as antispasmodic, analgesic, disinfectant, bactericidal, cicatrising, choleretic, tanning and decongestant, unnecessary surgical interventions were prevented.

Citation: Mufazalov F, Sufiyarov I, Hasanov A, Yamalova G, Bakirov E, Samorodov A. Spiral Computed Tomography with Phytocomposition as a Diagnostic Tool for Adhesive Intestinal Obstruction. J Biomed Phys Eng. 2020;10(5):607-612. doi: 10.31661/jbpe.v0i0.1912-1032.

\section{Keywords}

Intestinal Obstruction; Enema; Abdomen; Tanning; Analgesic; Phytocomposition

\section{Introduction}

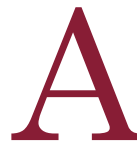
dhesive intestinal obstruction is considered one of the most common and potentially lethal complications after surgical interventions in the abdomen. According to the International Adhesion Society, the post-operative adhesive process in the abdomen is considered as a common complication of surgical interventions. Owing to peritoneal commissures, approximately $1 \%$ of previously operated
${ }^{1} \mathrm{PhD}$, Department of Oncology, Bashkir State Medical University, Ufa, Russia

${ }^{2} \mathrm{PhD}$, Department of Surgical Diseases,

Bashkir State Medical

University, Ufa, Russia

${ }^{3} \mathrm{MD}$, PhD Candidate,

Bashkir State Medical

University, Ufa, Russia

${ }^{4} \mathrm{PhD}$, Department of

Anesthesiology, Bashkir

State Medical University, Ufa, Russia

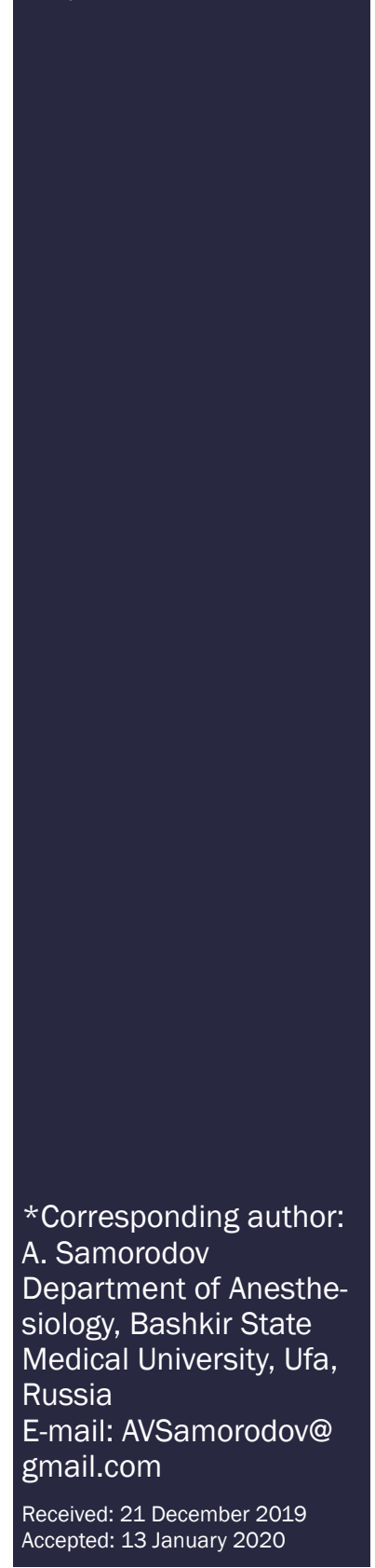


patients receive surgical treatment annually; of them, in $50 \%-75 \%$, acute adhesive intestinal obstruction develops, and mortality rate owing to this condition ranges from $13 \%$ to $55 \%$ [1].

Diagnosis of intestinal obstruction is complex concern in radiologic imaging. It is especially difficult to be diagnosed owing to the presence of a conglomerate of intestinal loops; generally, in a massive adhesion, a precise diagnosis cannot be made either by visual or densitometric analysis. Radiologic imaging plays a leading role in the diagnosis of intestinal obstruction, and the competent use of which in the vast majority of cases helps in confirming or excluding this pathology, determining the degree and nature of changes and ascertaining the cause of obstruction [2-4].

\section{Material and Methods}

In multidirectional cohort study, data was obtained with non-parallel (historical) control. This study included the analysis of results of patients with a diagnosis of intestinal obstruction $(n=50)$ who were admitted to the surgical departments of the City Clinical Hospitals No. 13 and No. 8 of Ufa city from 2013 to 2019; the patients' examination methods included computed tomography with conventional enhancement. The objective of this study was to analyse the diagnostic value of the spiral computed tomography performed with a novel method $(n=54)$. To conduct the study, permission was obtained from the ethics committee of the Federal State Budgetary Educational Institution of Higher Education at the Bashkir State Medical University under the Ministry of Health of Russian Federation. All patients provided written informed consent before participating in the study.

The proposed novel enhancing method was implemented as follows: the patient received oral mixture, containing $50 \mathrm{ml}$ of the contrast Unigexol $(300 \mathrm{mg})$ in $1 \mathrm{~L}$ cold mineral carbonated water, and CT was conducted within 40 min of ingesting the contrast meal. Further, the patient with suspected obstruction in the colon was administered a pre-prepared contrast enema with a decoction of leaves of smoke-tree $(100 \mathrm{~g})$, chamomile flowers (100 g) and calendula flowers $(100 \mathrm{~g})$.

Preparation method: the abovementioned herbal combination was dowsed with 1000 $\mathrm{ml}$ water (water absorption coefficient was considered) at room temperature, boiled in a water bath at $45^{\circ} \mathrm{C}$ and the prepared decoction was filtered through a 4-layer gauze fabric. The decoction was prepared from the same proportion, boiled for $15 \mathrm{~min}$, ingrained for $45 \mathrm{~min}$ and then filtered. Further, computed tomography was performed in the following order: the first, scan was panoramic, in which the general condition of the abdominal organs was assessed and a pathological focus was determined. The second, a series of scans was performed in a horizontal plane at the level of the detected changes (with a slice thickness of 0.5-5 mm). A 3D model was developed [Figure 1] using the Vitrea Advanced Visualization software (Canon Medical Systems Limited, Japan), and the presence of dilated intestinal loops, the inner and outer diameters of the small intestine, the thickness of the small intestine, the nature of the intestinal content, the occurrence of infiltrates in the abdominal cavity, and the presence of fluid in the abdominal cavity were determined [Figure 2]. This method has been patented as 'method for enhancing the colon at spiral computed tomography' (patent No. 2017129424).

The study results were analysed by statistical package Statistica 10,0 (StatSoft Inc, USA). Normality of the distribution of actual data was tested using the Shapiro-Wilk test. Comparison of categorical variables was performed using the $\chi^{2}$ test or Fisher's exact test. A p-value equals to 0.05 was considered statistically significant.

\section{Results}

A total of 54 patients were examined using the proposed method. In all the cases, the in- 


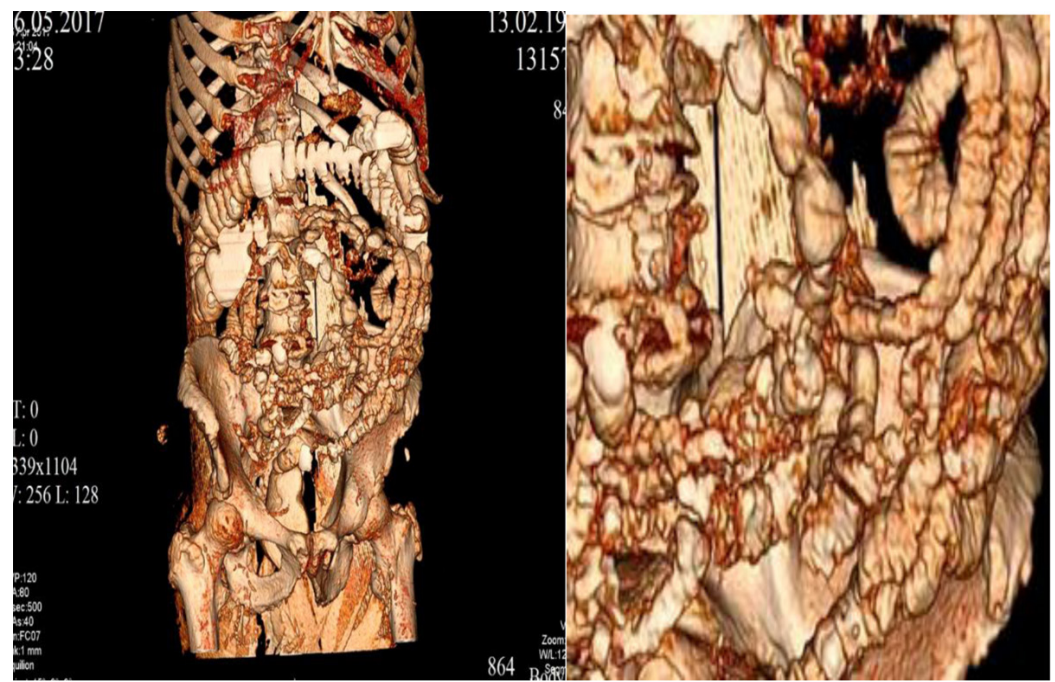

Figure 1: Spiral computed tomography in a patient with peritoneal commissures, with large bowel enhancement using a herbal mixture: a decoction of leaves of smoke-tree (100 g), chamomile flowers $(100 \mathrm{~g})$ and calendula flowers $(100 \mathrm{~g})$; 3D reconstruction in which deformation of the loops of the small intestine by the adhesive process is observed.

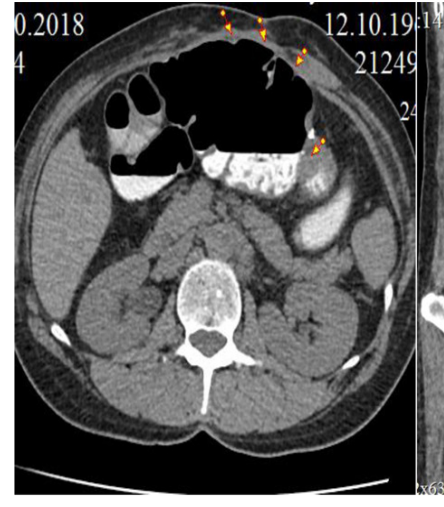

A

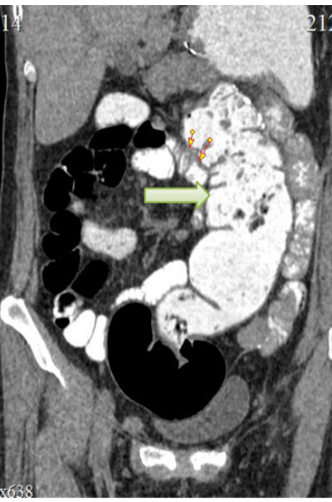

B

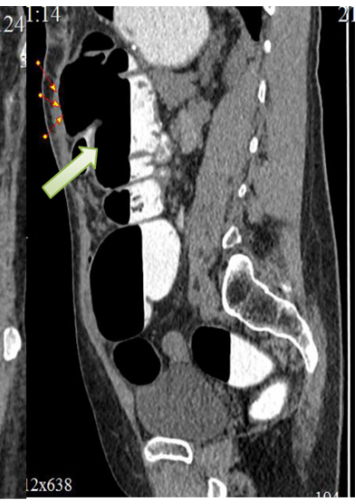

$\mathrm{C}$

Figure 2: Spiral computed tomography in a patient with peritoneal commissures with large bowel enhancement using a herbal mixture: a decoction of leaves of smoke-tree (100 g), chamomile flowers (100 g) and calendula flowers (100 g). Computed tomography reconstruction, arrows show adhesion sites: (A) axial, (B) coronar and (C) sagital projections.

formativeness of the examination was high, the diagnosis was accurate, and no complications were identified.

These results are supported with comparison of 2 groups of patients: in the first group $(n=54)$, the enhancing was performed with the proposed method, and in the second group $(\mathrm{n}=50)$, conventional computed tomography was performed [Table 1].

In addition, our study demonstrated that in the first group of patients, there were no allergic complications; however, in the second group, in which only an iodine-containing drug was used as an enema, $3(6 \%)$ patients had complications in the form of allergic reactions. 
The use of CT enterography with phytocomposition in patients of the first group and conventional water-soluble contrast of the second comparison group prevented unnecessary surgical intervention in $24(44.4 \%)$ patients of the first group and $19(38.0 \%)$ patients of the second group in which the obstruction was conservatively stopped [Table 2].

Consequently, the study allowed accurate diagnosis of patients, indicating an adequate treatment, particularly excluding the acute adhesive intestinal obstruction. There were no complications during the manipulation.

\section{Discussion}

The experience gained over several decades demonstrates the high value of the radiographic contrast study (RCS) of the intestine in the diagnosis of intestinal obstruction. Various methods of artificial enhancing have been developed and indications for their use have been clearly formulated depending on the anatomical and physiological characteristics of the examined site and the presence of particular clinical manifestations $[1,5]$.

The earliest and most physiological method of RCS of the small intestine is enhancement through an aqueous suspension of barium sul- phate which is orally ingested; this was first proposed in 1911 by G. Schwartz.

Currently, according to different literature data, oral RCS is rarely used owing to its insufficient informativeness duration of the process and high radiation exposure [5-7]. In addition, the majority of authors are unanimous that this technique should be completely excluded from the diagnostic arsenal when examining patients with any intestinal pathology as it is uninformative and, in some cases, harmful and even dangerous (for example, conversion of partial colonic obstruction is completed owing to the blockage of the narrowed part of the intestine by the hardened barium) $[1,5,8]$.

The positive feature of the ultrasound examination is the determination of access for laparoscopic surgeries; simultaneously, the authors note that this method is accessory and cannot substitute computed tomography. The authors consider that the advantage of computed tomography, unlike ultrasound, is its independence from the researcher; it is fast and does not require barium and has high informativeness $[1,5,8]$.

$\mathrm{X}$-ray computed tomography has a substantial advantage over traditional X-ray examination with a high sensitivity, which in the adhe-

Table 1: The effectiveness of enhancing by Computed tomography (CT) data with the stated method (group 1) and with the conventional computed tomography (group 2).

\begin{tabular}{cccc} 
Groups & Precision & Sensitivity & Specificity \\
\hline First group & ${ }^{*} 98.0(93.8-99.8)$ & ${ }^{*} 95.1(91.4-98.9)$ & $96.2(91.6-99.0)$ \\
\hline Second group & $82.6(75.8-86.5)$ & $83.1(77.8-89.5)$ & $84.7(78.8-88.3)$
\end{tabular}

* The differences are statistically significant compared with the secondgroup at $\mathrm{p}<0.05$, in parentheses- $-95 \%$ confidence interval.

Table 2: Number of patients treated conservatively and operatively in all study groups (\%).

\begin{tabular}{cccc} 
Type of treatment & First group $(\mathbf{n}=\mathbf{5 4}) \%$ & Second group $(\mathbf{n}=\mathbf{5 0}) \%$ & $\mathbf{P}$ \\
\hline Conservative & $24 / 44.4$ & $19 / 38.0$ & $X^{2}=5.92 ; \mathrm{df}=2 ; p=$ \\
\cline { 1 - 3 } Operative & $30 / 55.6$ & $31 / 62.0$ & 0.052
\end{tabular}


sive process in the abdominal cavity allows the determination of the location of the pathological conglomerate of intestinal loops, whereas the usual barium passage allows establishing the topography of the lesion in a single, frontal plane [1,3-5,8].

A panoramic X-ray is informative only in $50 \%-60 \%$ of cases; the passage of barium in peritoneal commissures is lengthy, and barium can cause obstruction of the intestine $[5,6,9,10]$.

Regarding ultrasound examination, the authors believe that the positive aspects are simplicity, low cost, minimal invasiveness, effectiveness and the absence of radiation exposure. There is a possibility of real-time assessment of the structures' motility. In their opinion, the sensitivity of the method is $69 \%$ $98 \%$. The use of ultrasound is limited by intestinal hyperpneumatisation. In addition, the method does not allow differentiating colon tumours with dimensions of $<3-4 \mathrm{~cm}$ in diameter $[1,4,7,8,11]$.

Compared with ultrasound examination, $\mathrm{X}$ ray computed tomography allows the documentation of pathological alterations in the abdominal cavity and liberates the surgeon from the need to be present at the diagnostic stage, i.e., considerably increases the degree of liberty and thus reduces labour costs $[6,7,12]$.

One of the modern evaluation methods is magnetic resonance tomography; it can be used to image any part of the body without ionising radiation. It is highly informative and specific $[6,8,13,14]$. This involves excellent visualisation of the abdominal organs without contrast enhancement; however, the duration of the study with permanent breath holdings is not acceptable for patients with extreme pain syndrome and severe general condition $[15,16]$.

The most objective and informative method for the examination of intestinal adhesions is laparoscopy; however, it is an invasive research method associated with possible damage to the intestine during the induction of pneumoperitoneum and relapse of the adhesive process $[9,10,11,15,17]$.

Therefore, compared with other imaging methods, X-ray computed tomography, with multiplanar reconstruction and 3D structuring, allows to optimally improve the precision of the topographic anatomical assessment of the pathological focus and to determine the possibility of a surgical treatment method. The proposed enhancement method is easily reproducible in a hospital setting; a technical result is achieved using this method, and it meets the criterion of 'economic usefulness'. One drawback of CT is radiation exposure.

Consequently, the success of the diagnosis of peritoneal commissures is determined by the integrated use of clinical and instrumental methods of examination.

\section{Conclusion}

Owing to early diagnosis of intestinal obstruction and application of the phytocomposition during the examination, exerting effects such as antispasmodic, analgesic, disinfectant, bactericidal, cicatrising, choleretic, tanning and decongestant, unnecessary surgical intervention was prevented in patients and obstruction was conservatively stopped in 24 (44.4\%) patients in the main group. The remaining 30 (55.6\%) patients from the main group were operated with minimal surgical access in the early stages.

\section{Conflict of Interest}

None

\section{References}

1. Gumán-Valdivia-Gómez G, Tena-Betancourt E. Postoperative abdominal adhesions: pathogenesis and current preventive techniques. Cir Cir. 2019;87(6):698-703. doi: 10.24875/ CIRU.18000511. PubMed PMID: 31631189.

2. Balthazar EJ, Birnbaum BA, Megibow AJ, Gordon RB, Whelan CA, Hulnick DH. Closed-loop and strangulating intestinal obstruction: CT signs. Radiology. 1992;185(3):769-75. doi: 10.1148/radiology.185.3.1438761. PubMed PMID: 1438761. 
3. Millet I, Boutot D, Faget C, Pages-Bouic E, Molinari N, Zins M, Taourel P. Assessment of strangulation in adhesive small bowel obstruction on the basis of combined CT findings: implications for clinical care. Radiology. 2017;285(3):798-808. doi: 10.1148/radiol.2017162352. PubMed PMID: 28759326.

4. Nakashima K, Ishimaru H, Fujimoto T, Mizowaki T, Mitarai K, Nakashima K, Matsuoka Y, Uetani $M$. Diagnostic performance of CT findings for bowel ischemia and necrosis in closed-loop small-bowel obstruction. Abdominal Imaging. 2015;40(5):1097-103. doi: 10.1007/s00261-0140335-2. PubMed PMID: 25542218.

5. Rondenet C, Millet I, Corno L, Boulay-Coletta I, Taourel P, Zins M. Increased unenhanced bowelwall attenuation: a specific sign of bowel necrosis in closed-loop small-bowel obstruction. European Radiology. 2018;28(10):4225-33. doi: 10.1007/ s00330-018-5402-6. PubMed PMID: 29679213.

6. Millet I, Taourel P, Ruyer A, Molinari N. Value of CT findings to predict surgical ischemia in small bowel obstruction: a systematic review and metaanalysis. European Radiology. 2015;25(6):182335. doi: 10.1007/s00330-014-3440-2. PubMed PMID: 25850889.

7. Khaled W, Millet I, Corno L, Bouley-Coletta I, Benadjaoud MA, Taourel P, Zins M. Clinical relevance of the feces sign in small-bowel obstruction due to adhesions depends on its location. American Journal of Roentgenology. 2018;210(1):78-84. doi: 10.2214/AJR.17.18126. PubMed PMID: 29045179.

8. Cohen JF, Korevaar DA, Altman DG, Bruns DE, Gatsonis CA, Hooft L, Irwig L, Levine D, Reitsma JB, De Vet HC, Bossuyt PM. STARD 2015 guidelines for reporting diagnostic accuracy studies: explanation and elaboration. BMJ Open. 2016;6:e012799. doi: 10.1136/bmjopen-2016-012799.

9. Chen $Q$, Jiang J. Recognition of the diagnosis and treatment of small intestinal obstruction. Chinese Journal of Gastrointestinal Surgery. 2017;20(10):1136-40. PubMed PMID: 29130227.

10. Wieland WF, Burger M, Denzinger S, Otto W, Pavlov VN. Radical Prostatectomy: from Open
Surgery towards Robotic Laparoscopy. Creative Surgery and Oncology. 2020;10(2):87-93. doi: 10.24060/2076-3093-2020-10-2-87-93.

11. Pujahari AK. Decision making in bowel obstruction: a review. JCDR. 2016;10(11):PE07. doi: 10.7860/JCDR/2016/22170.8923. PubMed PMID: 28050445. PubMed PMCID: PMC5198398.

12. Sekiba K, Ohmae T, Odawara N, Moriyama M, Kanai S, Tsuboi M, Saito T, Uchino K, Akamatsu $M$, Okamoto M. A new method for insertion of long intestinal tube for small bowel obstruction: Nonendoscopic over-the-wire method via short nasogastric tube. Medicine. 2016;95(47). doi: 10.1097/md.0000000000005449. PubMed PMID: 27893689. PubMed PMCID: PMC5134882.

13. Khasanov AG, Sufiyarov IF, Bakirov ER, Nurtdinov MA, Ibraev AV, Evdokimov EV. Acute Small Intestinal Obstruction Caused by Ectopic Pancreas. A Clinical Case Report. Creative Surgery and Oncology. 2019;9(1):76. doi: 10.24060/2076-30932019-9-1-75-79.

14. Menys A, Butt $S$, Emmanuel A, Plumb AA, Fikree A, Knowles C, Atkinson D, Zarate N, Halligan S, Taylor SA. Comparative quantitative assessment of global small bowel motility using magnetic resonance imaging in chronic intestinal pseudoobstruction and healthy controls. Neurogastroenterology \& Motility. 2016;28(3):376-83. doi: 10.1111/nmo.12735.

15. D’Arpa F, Orlando G, Tutino R, Salamone G, Battaglia EO, Gulotta G. Conservative management of intestinal obstruction by isolated intramural duodenal hematoma. A case report and review of the literature. Ann Ital Chir. 2015;86(ePub):S2239253 X1502410X. PubMed PMID: 26675664.

16. Zhu W. Prevention and management of intestinal obstruction after gastrointestinal surgery. Chinese Journal of Gastrointestinal Surgery. 2016;19(4):376-8. PubMed PMID: 27112465.

17. Timerbulatov SV, Timerbulatov VM, Sagitov RB, Mekhdiev DI, Sakhautdinov RM. Acute Appendicitis: Clinical Laboratory, Laparoscopic, Pathomorphological Parallels. Creative Surgery and Oncology. 2019;9(1):13-7. doi: 10.24060/20763093-2019-9-1-13-17. 